\title{
Estudos climatológicos da Reserva Florestal Ducke - Manaus-AM.
}

\author{
IV. Precipitação (1)
}

\author{
Ari de Oliveira Marques Filho ${ }^{2}$ \\ Maria de Nazaré Góes Ribeiro $\left.{ }^{2}\right]$ \\ Hugo Menezes dos Santos ( $\left.{ }^{3}\right)$ \\ Jesus Marden dos Santos [ ${ }^{2}$ ?
}

\section{Resumo}

Neste trabalho, foi estudada a relação entre intensidade-freqüência-duração das precipitações ocorridas desde 1965 na Estação Climatológica Principal da Reserva Florestal Ducke, pertencente ao Instituto Nacional de Pesquisas da Amazônia, localizada no Km 26 da Rodovia Torquato Tapajós. A distribuição de freqüência proposta por Gumbel e uma distribuição exponencial foram ajustadas às Séries de Intensidades Máximas Anuais e Séries Parciais formadas. Determinou-se o grau de ajustamento dessas distribuiçōes aos dados observados para precipitaçōes com duração de 10 minutos.

\section{INTRODUÇÃO}

O conhecimento de métodos simplificados para a determinação de descargas, seja nos ios seja em sistemas de galerias pluviais em centros urbanos, permite a determinação de cheias críticas que devem ser consideradas nos projetos de obras hidráulicas, desde que sejam conhecidas as características das precipitações que provocam as situações limites em cada projeto.

Esse contexto mostra a importância de estudos que relacionam intensidade-freqüênciaduração das precipitações.

Uma análise ampla de chuvas intensas para diversas cidades brasileiras foi realizada por Pfafstetter (1957). Outros exemplos de estudos dessa natureza são encontrados na publicação de Garcez (1974), onde aparecem reproduzidos os trabalhos do Professor Parigot de Souza scbre precipitações em Curitiba e dos Engenheiros U. Alcântara e A. Rocha Lima que analisaram as chuvas intensas ocorridas no Rio de Janeiro.

Este trabalho apresenta algumas características semelhantes a esses estudos, e foi completamente com informações condensadas no Natural Environment Research Council (1975).

\section{MATERIAL E MÉTODOS}

Na Estação Climatológica da Reserva Florestal Ducke do Instituto Nacional de Pesquisas da Amazônia, localizada no $\mathrm{Km} 26$ da Rodovia Torquato Tapajós, foram coletados dados de precipitação, através de pluviógrafo tipo Heilmann-95, correspondentes ao período de abril de 1965 a novembro de 1980 .

Foram estudadas a Série de Intensidades Máxirnas Anuais e um caso particular de Série Parcial de Precipitações, na qual o número de elementos foi tomado igual ao número de anos de observações.

Período de retorno, T, é o período de tempo médio, expresso em anos, em que um determinado evento deve ser igualado ou superado pelo menos uma vez. Definindo-se $F(x)$ como a probabilidade de um evento $X$ ser menor ou igual a $x$, tem-se

$$
\mathrm{F}(\mathrm{x})=\operatorname{Pr}(\mathrm{X} \leq \mathrm{x})
$$

e o período de retorno pode ser representado por

$$
T=\frac{1}{1-F(x)}
$$

(1) - Trabalho inteiramente subvencionado pelo Conselho Nacional de Desenvolvimento Científico e Tecnológico (CNPq).

(2) - Instituto Nacional de Pesquisas da Amazônia, Manaus.

(3) - Instituto de Tecnologia do Amazonas, Manaus. 
Uma variável aleatória é caracterizada pela sue distribuição de probabilidades $F(x)$. As$\operatorname{sim}$, as distribuições de freqüências usadas serão estudadas, a partir dessas ligações entre o período de retorno e a probabilidade de ocorrência de determinado evento.

A distribuição de freqüência proposta por Gumbel aplica-se a séries hidrológicas compostas por valores extremos (Séries dos Máximos Anuais) e pode ser apresentada na seguinte forma matemática :

$F(x)=\frac{T-1}{T}=e^{-\theta-}\left(\gamma+\frac{x-\mu}{\sigma} \cdot \frac{\pi}{\sqrt{ } 6}\right)_{(2)}$

once,

$T$ - período de retorno da variável $x$.

$x$ - evento máximo anual.

$\mu$ - média dos eventos máximos anuais.

$\sigma$ - desvio padrão dos eventos máximos anuais.

$\gamma-$ constante de Euler $\simeq 0,5772$.

Segundo Chow (1964), é possivel resolver a maioria das funções de freqüências, usadas em Hidrologia, pela expressão

$$
\mathrm{x}=\mu+\mathrm{K} \sigma
$$

onde,

$$
\begin{aligned}
& \mathrm{K} \text { - fator de frequiência característico de } \\
& \text { cada óistribuição. }
\end{aligned}
$$

A equação (2) pode ser transformada para uma forma semeihante à equação (3), e o fator de freqüência, neste caso, fica dado pela expressão

$$
K=-\frac{V^{\prime} 6}{\pi}\left[\operatorname{Ln} \operatorname{Ln} \frac{T}{T-1}+\gamma\right]
$$

A distribuição exponencial pode adaptar-se a séries formadas pelos valores superiores a um valor básico, xo. embora isto deva ser comprovado pelo seu ajustamento às observações (Natural Environment Research Council, 1975).

A distribuição condicional das séries parciais, quando descrita na forma exponencial, apresenta o seguinte aspecto:

$$
F\left(X \leqslant x \mid x \geqslant x_{0}\right)=1-e^{-(x-x) / \beta}
$$

onde,

$F($ ) - é a probabilidade que um evento $X$ seja $\leqslant x$, quando $x \geqslant x_{0}$.

$x \quad$ - evento aleatório.

$\mathrm{x}_{0}$ - valor básico.

î - parâmetro.

A relação da probabilidade com o período de retorno está implícita na equação (5), sendo dada por

$$
F\left(X \leqslant x \mid x \geqslant x_{0}\right)=1-\frac{1}{\lambda T}
$$

onde,

$\lambda$ - Número médio de valores que excedem $x_{0}$ no período de um ano.

No caso particular onde se fixa o número de eventos que formarão a série, designado aqui por $\mathrm{M}$, o valor de $\lambda$ é dado pela razão

$$
\lambda=\mathrm{M} / \mathrm{N}
$$

onde,

$\mathrm{N}$ - números de anos de observações.

Quando se procede dessa maneira, o valor básico $x_{0}$ não pode ser determinado sem relacioná-lo aos dados observados. Esse valor deve ser estimado estatisticamente. Da mesma forma, deve ser obtida uma estimativa do parâmetro $\beta$ da distribuição exponencial.

As estimativas desses dois valores pelo método da máxima verossimilhança são dadas pelas seguintes expressões

$$
\begin{aligned}
& \hat{x}_{0}=\frac{M \cdot X_{\min }-\bar{x}}{M-1} \\
& \hat{\beta}=\frac{M\left(\bar{x}-X_{\min }\right)}{M \cdot 1}
\end{aligned}
$$

As estimativas dos valores esperados para um dado período de retorno $T$ são dados pela expressão

$$
x=\hat{x}_{0}+\hat{\beta}(\operatorname{Ln} \lambda+\operatorname{Ln} T)
$$

Nas relações acima $x_{\min }$ é o menor valor dos eventos constituintes da série relacionada, e $\bar{X}$ é a média desses eventos. 
As séries de precipitações máximas (ou intensidades máximas) precisam ser selecionadas, segundo a duração de cada evento, para que se possa introduzir essa variável numa equação final de intensidade-freqüência-duração.

A relação entre as intensidades, estimadas para diferentes períodos de retorno, e a duração de uma precipitação é pesquisada usualmente através da fórmula do tipo

$$
i=\frac{a}{\left(t+t_{0}\right) n}
$$

onde,

$\mathrm{i}$ - intensidade da chuva em $\mathrm{mm} / \mathrm{h}$.

$t$ - duração da chuva em minutos.

a, $t_{0}, \mathrm{n}$ - parâmetros que devem ser determinados.

As intensidades versus durações lançadas em gráficos logarítmicos, para um mesmo período de retorno, ajustam-se a uma curva convexa. A constante adicionada a cada duração de precipitação, que transforma essa curva numa reta, é uma estimativa do parâmetro $t_{0}$. Os valores dos parâmetros a e $n$ estão relacionados $\mathrm{com}$ os coeficientes linear e angular dessa reta, respectivamente.

As curvas correspondentes aos diversos períodos de retorno apresentam conformações bem semelhantes, e, ao serem transformadas em retas, essa semelhança fica mantida no paralelismo aproximado das mesmas. Valendose disso, podem ser obtidas diversas equações semelhantes à equação 10 , que apresentam como elemento distinto apenas o parâmetro $a$, diferente para cada período de retorno considerado.

Com os pares de vaiores a e $T$, pesquisouse uma relação da forma

$$
\mathrm{a}=\mathrm{cTm}
$$

onde $c$ e $m$ são parâmetros determinados através do método dos mínimos quadrados.

A expressão que relaciona intensidade-freqüência-duração das precipitações apresenta-se na forma

$$
i=\frac{c T m}{\left(t+t_{0}\right)^{n}}
$$

\section{RESUltados OBTIDOS E DISCUSSÃo}

Os totais mensais de precipitaçōes observadas no período de abril de 1965 a novembro de 1980 são apresentados na tabela 1.

$\mathrm{Na}$ figura 1, são apresentados os valores médios, máximos e mínimos para cada mês. $\mathrm{O}$ período chuvoso estende-se desde novembro até o mès de maio, e nos meses restantes ocorre uma queda sensivel nas precipitações. notadamente nos meses de agosto e setembro.

Esse caráter cíclico, bem definido, da dis. tribuição de precipitação ao longo do ano explica assim o relativo sucesso da simulação dessas variações através de modelos matemáticos.

O registro das precipitações nessa Estação Meteorológica apresenta falhas apenas em alguns meses dos anos de 1971 e 1972 e o período total de informações corresponde a 15 anos. Esses dados permitiram o estudo de intensidade-freqüência-duração das precipitações.

A forma de seleção dos eventos para um estudo sobre chuvas intensas está inserida nos procedimentos usuais em análises desse tipo. Assim, na série de observações, foram escolhidas as precipitações mais significativas estabelecendo-se, antecipadamente, limites para que uma chuva de dada duração fosse considerada. Esses limites mínimos são 10, 12. $15,20,25,28,30,35$ e $45 \mathrm{~mm}$ para as durações de $5,10,15,20,30,45,60,90$ e 120 minutos, respectivamente. Com esses limites, o número de precipitações ficou reduzido aos eventos que tinham alturas máximas suficientes para serem incluídas nas séries formadas.

O elevado número de precipitações, 103, indicou que os limites mínimos escolhidos foram baixos. A definição a priori desses limites näo é simples, devendo sofrer adaptações, no desenrolar do estudo.

A tabela 2 mostra as séries de intensidades máximas anuais já dispostas em ordem decrescente das magnitudes para cada duração. A tabela 3 apresenta as séries dos quinze eventos máximos do período, disposta na mesma forma da tabela anterior.

Sobre esses elementos, foram aplicados os procedimentos anteriormente descritos. 


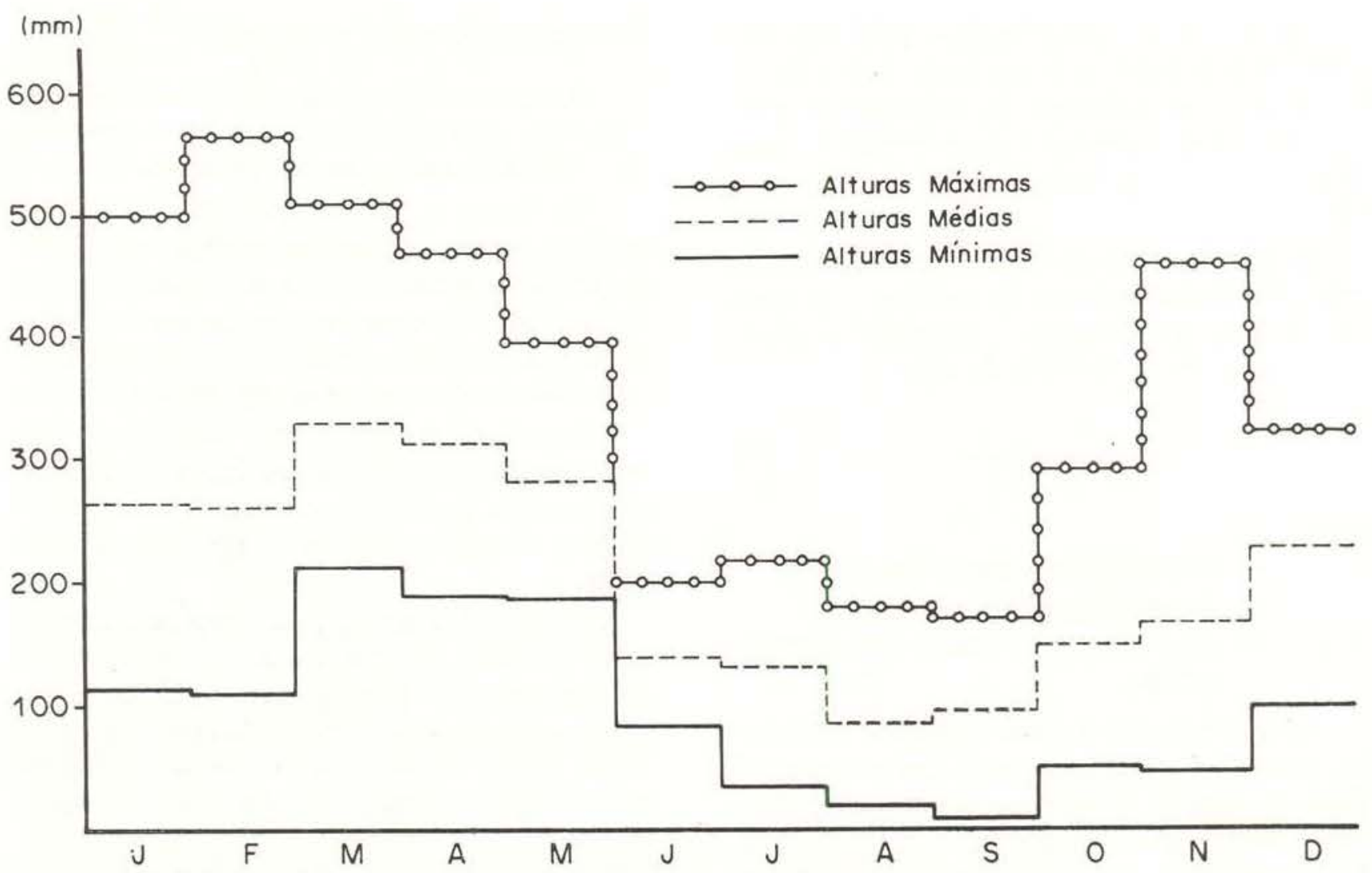

Fig. 1 - Precipitações mensais médias, máximas e mínimas.

TABELA 1 - Totais Mensais e Anuais de Precipitação

\begin{tabular}{|c|c|c|c|c|c|c|c|c|c|c|c|c|c|}
\hline IVI & Jan. & Fev. & Mar. & Abr. & Mai. & Jun. & Jul. & Ago. & Set. & Out. & Nov. & Dez. & Totais \\
\hline 1965 & & & & 306,0 & 208,6 & 117,9 & 118,4 & 16,5 & 41,4 & 90,9 & 111,9 & 310,2 & 1321,8 \\
\hline 1966 & 308,0 & 243,6 & 212,1 & 212,7 & 396,9 & 109,5 & 198,5 & 94,2 & 167,9 & 74,0 & 252,1 & 185,9 & 2455,4 \\
\hline 1967 & 343,7 & 245,8 & 328,8 & 374,2 & 185,5 & 92,7 & 31,3 & 47,4 & 54,6 & 88,6 & 179,5 & 201,6 & 2173,7 \\
\hline 1968 & 248,7 & 161,3 & 314,0 & 469,8 & 380,1 & 122,4 & 102,9 & 178,0 & 155,2 & 107,5 & 459,6 & 204,9 & 2904,4 \\
\hline 1969 & 224,2 & 253,8 & 343,4 & 392,3 & 280,7 & 81,6 & 121,5 & 130,2 & 15,2 & 162,3 & 43,3 & 292,5 & 2341,0 \\
\hline 1970 & 350,2 & 386,4 & 226,9 & 301,4 & 193,8 & 152,6 & 164,0 & 70,1 & 103,1 & 47,5 & 162,2 & 171,7 & 2329,9 \\
\hline 1971 & 308,2 & 402,9 & 509,9 & 322,0 & 332,8 & 184,2 & 95,1 & 63,2 & 55,6 & 234,6 & 138,0 & 97,8 & 2744,3 \\
\hline 1972 & 262,8 & 341,4 & 290,5 & 267,8 & 285.4 & 158,6 & 191,4 & 99,4 & 52,8 & 184,2 & 93,2 & 210,0 & 2437,5 \\
\hline 1973 & 212,9 & 194,7 & 420,1 & 194,0 & 392.1 & 197,4 & 215,8 & 160,8 & 136,1 & 112,7 & 146,2 & 322,1 & 2704,9 \\
\hline 1974 & 142,0 & 248,3 & 264,5 & 337,1 & 271,5 & 181,8 & 180,4 & 73,2 & 114,6 & 47,6 & 209,8 & 202,8 & 2273.6 \\
\hline 1975 & 228,3 & 186,1 & 269,9 & 321,0 & 305,3 & 134,3 & 121,0 & 88,2 & 40,5 & 290,7 & 159,7 & 216,6 & 2371,6 \\
\hline 1976 & 307,6 & 568,2 & 401,8 & 312,0 & 209,4 & 89,5 & 124,1 & 51,8 & 6,8 & 203,0 & 77,4 & 262,7 & 2614,3 \\
\hline 1977 & 114,0 & 225,2 & 381,8 & 391,2 & 267,8 & 98,4 & 79,9 & 23,0 & 165,8 & 275,8 & 149,6 & 232,4 & 2434,9 \\
\hline 1978 & 274,5 & 218,5 & 376,6 & 334,1 & 278,0 & 139,3 & 133,8 & 41,8 & 167,0 & 85,1 & 70,6 & 155,4 & 2274,7 \\
\hline 1979 & 4980 & 144,1 & 380,8 & 283,6 & 323,5 & 128,8 & 55,3 & 135,6 & 122,1 & 182,3 & 200,5 & 287,3 & 2741,9 \\
\hline 1980 & 137,6 & 111,0 & 230,8 & 187,5 & 236,1 & 188,8 & 100,7 & 47.4 & 86,2 & 148,0 & 190,7 & & 1664,8 \\
\hline$\Sigma$ & 3960,7 & 3931,3 & 4951,9 & 5006,7 & 4547,5 & 2177,8 & 2034,1 & 1320,8 & 1484,9 & 2334,8 & 2654,3 & 3383,9 & 37788,7 \\
\hline Média & 264,0 & 262,1 & 330,1 & 312,9 & 284,2 & 136,1 & 127,1 & 82,6 & 92,8 & 145,9 & 165,9 & 225,6 & \\
\hline
\end{tabular}




\begin{tabular}{|c|c|c|c|c|c|c|c|c|c|c|c|c|c|c|c|c|c|c|}
\hline & & & & & & TAB & $42-$ & éries & as Intens & dades & iximas & wais & & & & & & \\
\hline \multirow{2}{*}{$\begin{array}{c}\text { Dur. } \\
\mathrm{N} \text {. }\end{array}$} & \multicolumn{2}{|c|}{$5 \mathrm{~min}$} & \multicolumn{2}{|c|}{$10 \mathrm{~min}$} & \multicolumn{2}{|c|}{$15 \mathrm{~min}$} & \multicolumn{2}{|c|}{$20 \mathrm{~min}$} & \multicolumn{2}{|c|}{$30 \mathrm{~min}$} & \multicolumn{2}{|c|}{$45 \mathrm{~min}$} & \multicolumn{2}{|c|}{$60 \mathrm{~min}$} & \multicolumn{2}{|c|}{$90 \mathrm{~min}$} & \multicolumn{2}{|c|}{$120 \mathrm{~min}$} \\
\hline & Data & $\mathrm{mm} / \mathrm{l}$ & Data & $\mathrm{mm} / \mathrm{t}$. & Data & $\mathrm{mm} / \mathrm{h}$ & Data & $\mathrm{mm} / \mathrm{h}$ & Data & $\mathrm{mm} / \mathrm{h}$ & Data & $\mathrm{mm} / \mathrm{h}$ & Data & $\mathrm{mm} / \mathrm{h}$ & Data & $\mathrm{mm} / \mathrm{h}$ & Data & $\mathrm{mm} / \mathrm{h}$ \\
\hline 1 & 13.02 .70 & 240,0 & 13.02 .70 & 168,0 & 13.02 .70 & 140,0 & 21.04 .68 & 121,5 & 21.04 .68 & 115,0 & 21.04 .68 & 104,3 & 07.03 .78 & 97,5 & 07.03 .78 & 89,8 & 07.03 .78 & 72,9 \\
\hline 2 & 23.03 .80 & 204,0 & 23.03 .80 & \begin{tabular}{|l|}
146,4 \\
\end{tabular} & 21.04 .68 & 128,0 & 13.02 .70 & 120,0 & 27.12 .76 & 108,0 & 25.10 .75 & 91,3 & 21.04 .68 & 88,6 & 21.04 .68 & 68,7 & 23.11.68 & 59,3 \\
\hline 3 & 05.01.79 & 171,6 & 18.12 .76 & 138,0 & 27.12.76 & \begin{tabular}{|l|}
128,0 \\
\end{tabular} & 02.04 .65 & \begin{tabular}{|l|}
117,0 \\
\end{tabular} & 27.10 .69 & 105,0 & 07.03 .78 & 91,3 & 25.10 .75 & 82,8 & 25.10 .75 & 60,3 & 27.10 .69 & 50,6 \\
\hline 4 & 27.12.76 & 168,0 & 21.04 .68 & 132,0 & 18.01 .66 & 124,4 & 18.01 .66 & 116.1 & 07.03 .78 & 105,0 & 27.10 .69 & 90,0 & 27.10.69 & 75,4 & 27.10 .69 & 56,7 & 07.02 .76 & 45,7 \\
\hline 5 & 27.12 .69 & 160,8 & 27.10 .69 & 132,0 & 02.04 .65 & 124,0 & 27.12 .76 & $\mid 114,0$ & 05.01 .79 & 102,6 & 27.12.76 & 86,1 & 16.11 .74 & 69,9 & 16.11 .74 & 47,9 & 25.10 .75 & 45,4 \\
\hline 6 & 25.10 .75 & 156,0 & 27.10 .75 & 132,0 & 10.01 .73 & 120,4 & 05.01 .79 & 111,9 & 13.02 .70 & 100,0 & 16.11 .74 & 79,9 & 24.03 .73 & \begin{tabular}{|l|}
66,8 \\
\end{tabular} & 31.03 .71 & 47,7 & 1601.70 & 42,2 \\
\hline 7 & 07.03.78 & 150,0 & 07.03.78 & 129,0 & 16.11 .74 & 120,0 & 16.11 .74 & 111,0 & 16.11 .74 & 93,8 & 24.03.73 & 79.5 & 27.12.76 & 64,6 & 16.01 .70 & 46,9 & 12.12 .73 & 38,3 \\
\hline 8 & 19.12 .67 & 144,0 & 18.01 .66 & \begin{tabular}{|l}
127,8 \\
\end{tabular} & 07.03.78 & 120,0 & 10.01 .73 & 109,8 & 02.04 .65 & 92,4 & 05.01 .79 & 77,7 & 31.03 .71 & 62,3 & 07.02 .76 & 46,6 & 31.03 .71 & 37,2 \\
\hline 9 & 21.04 .68 & 144,0 & 02.04 .65 & 123,0 & 05.01 .79 & 120,0 & 25.10 .75 & \begin{tabular}{|l}
109,5 \\
\end{tabular} & 25.10 .75 & 89,6 & 16.01 .70 & 75,1 & 05.01 .79 & 61,7 & 18.01 .66 & 46,1 & 16.11 .74 & 36,3 \\
\hline 10 & 14.05 .66 & 134,4 & 10.01 .73 & \begin{tabular}{|l}
121,8 \\
\end{tabular} & 25.10 .75 & 118,0 & 07.03.78 & \begin{tabular}{|l|}
109,5 \\
\end{tabular} & 18.01 .66 & 87,0 & 31.03 .71 & 73,7 & 16.01 .70 & 60,9 & 24.03 .79 & 45,9 & 18.01 .66 & 35,4 \\
\hline 11 & 10.01 .73 & 133,2 & 16.11 .74 & 120,0 & 23.03 .80 & 113,2 & 27.10 .69 & 100,5 & 27.07 .73 & 86,8 & 06.05 .77 & 67.5 & 18.01 .66 & 57.5 & 05.01 .79 & 41.9 & 16.04.77 & 31,6 \\
\hline 12 & 02.04 .65 & 132,0 & 05.01 .79 & 120,0 & 27.10 .69 & 112,0 & 29.09 .77 & 99,0 & 31.03 .71 & 80,0 & 18.01 .66 & 67,3 & 06.05 .77 & 57,4 & 06.05 .77 & 39,4 & 05.01 .79 & 31,6 \\
\hline 13 & 15.07 .74 & 132,0 & 19.12 .67 & 117,0 & 19.12 .67 & 104,0 & 19.12 .67 & 93,0 & 20.06 .80 & 80,0 & 20.06 .80 & 66,7 & 20.06 .80 & 54,5 & 20.06 .80 & 38,9 & 08.04.67 & 30,0 \\
\hline 14 & 29.09 .77 & 126,0 & 29.09.77 & 111,0 & 06.05 .77 & 104,0 & 31.03 .71 & 91,8 & 08.04.67 & 77,0 & 02.04 .65 & 66,3 & 08.04 .67 & 50,5 & 08.04 .67 & 36,3 & 20.06 .80 & 29,5 \\
\hline 15 & 15.01 .71 & 120,0 & 15.01 .71 & 102,0 & 31.03 .71 & 96,8 & 20.06 .80 & 91,8 & 29.09 .77 & 75,4 & 08.04 .67 & 63,1 & 02.04 .65 & 50,4 & 02.04 .65 & 33,6 & 02.04 .65 & 25,2 \\
\hline Média & - & 154,4 & - & \begin{tabular}{|l|}
128,0 \\
\end{tabular} & - & $\mid 118,2$ & - & \begin{tabular}{|l|}
107,8 \\
\end{tabular} & - & 93,2 & - & 78,7 & - & 66,7 & - & 49,8 & - & 40.7 \\
\hline 6 & - & 32,1 & - & 15,5 & - & 11,0 & - & 10,1 & - & 12,3 & - & 11,9 & - & 13,9 & - & 14,4 & - & 12,7 \\
\hline
\end{tabular}




\begin{tabular}{|c|c|c|c|c|c|c|c|c|c|c|c|c|c|c|c|c|c|c|}
\hline Dur. & \multicolumn{2}{|c|}{$5 \mathrm{~min}$} & \multicolumn{2}{|c|}{$10 \mathrm{~min}$} & \multicolumn{2}{|c|}{$15 \mathrm{~min}$} & \multicolumn{2}{|c|}{$20 \mathrm{~min}$} & \multicolumn{2}{|c|}{$30 \mathrm{~min}$} & \multicolumn{2}{|c|}{$45 \mathrm{~min}$} & \multicolumn{2}{|c|}{$60 \mathrm{~min}$} & \multicolumn{2}{|c|}{$90 \mathrm{~min}$} & \multicolumn{2}{|c|}{$120 \mathrm{~min}$} \\
\hline N. ${ }^{\circ}$ & Data & $\mathrm{mm} / \mathrm{h}$ & Data & $\mathrm{mm} / \mathrm{h}$ & Data & $\mathrm{mm} / \mathrm{h}$ & Data & $\mathrm{mm} / \mathrm{h}$ & Data & $\mathrm{mm} / \mathrm{h}$ & Data & $\mathrm{mm} / \mathrm{h}$ & Data & $\mathrm{mm} / \mathrm{h}$ & Data & $\mathrm{mm} / \mathrm{h}$ & Data & $\mathrm{mm} / \mathrm{h}$ \\
\hline 1 & 13.02 .70 & 240,0 & 13.02 .70 & 168,0 & 13.02 .70 & 140,0 & 21.04 .68 & 121,5 & 21.04 .68 & 115,0 & 21.04 .68 & 104,3 & 07.03.78 & 97,5 & 07.03 .78 & 89,8 & 07.03 .78 & 72,9 \\
\hline 2 & 23.03 .80 & 204,0 & 23.03 .80 & 146,4 & 21.04 .68 & 128,0 & 13.02 .70 & 120,0 & 29.10 .72 & 110,0 & 25.10 .75 & 91,3 & 21.04 .68 & 88,6 & 21.04 .68 & 68,7 & 23.11 .68 & 59,3 \\
\hline 3 & 05.01 .79 & 171,6 & 18.12 .76 & 138,0 & 27.12.76 & 128,0 & 29.10.72 & 120,0 & 27.12.76 & 108,0 & 07.03 .78 & 91,3 & 25.10 .75 & 82,8 & 23.11 .68 & 61,3 & 21.04 .68 & 52,1 \\
\hline 4 & 27.12.76 & 168,0 & 18.12 .72 & 135,0 & 06.05 .68 & 126,0 & 02.04 .65 & 117,0 & 27.10 .69 & 105,0 & 27.10 .69 & 90,0 & 27.10 .69 & 75,4 & 25.10 .75 & 60,3 & 27.10 .69 & 50,6 \\
\hline 5 & 27.12.79 & 160,8 & 21.04 .68 & 132,0 & 16.01 .70 & 126,0 & 18.01 .66 & 116,1 & 07.03 .78 & 105,0 & 27.12.76 & 86,1 & 23.11 .68 & 72,5 & 27.10 .69 & 56,7 & 07.02 .76 & 45,7 \\
\hline 6 & 25.10 .75 & 156,0 & 27.10 .69 & 132,0 & 18.01 .66 & 124,4 & 27.12.76 & 114,0 & 05.01 .79 & 102,6 & 29.10 .72 & 86,0 & 16.11 .74 & 69,9 & 16.11 .74 & 47,9 & 25.10 .75 & 45,4 \\
\hline 7 & 18.12 .76 & 156,0 & 27.10.75 & 132,0 & 02.04 .65 & 124,0 & 05.01.79 & 111,9 & 13.02 .70 & 100,0 & 16.11 .74 & 79,9 & 24.03 .71 & 66,8 & 31.03 .71 & 47,7 & 16.01 .70 & 42,2 \\
\hline 8 & 07.03.78 & 150,0 & 02.04 .65 & 132,0 & 10.01 .73 & \begin{tabular}{|l}
120,4 \\
\end{tabular} & 06.05 .68 & 111,9 & 16.11 .74 & 93,8 & 24.03 .73 & 79,5 & 29.10 .72 & 66,0 & 16.01 .70 & 46,9 & 16.04 .68 & 42,0 \\
\hline 9 & 19.12 .67 & 144,0 & 07.03 .78 & 129,0 & 16.11 .74 & 120,0 & 16.11 .74 & 111,0 & 23.11 .68 & 93,0 & 05.01 .79 & 77,7 & 27.12.76 & 64,6 & 07.02 .76 & 46,6 & 12.12 .73 & 38,3 \\
\hline 10 & 21.04 .68 & 144,0 & 06.05 .68 & 129,0 & 07.03.78 & 120,0 & 10.01 .73 & 109,8 & 02.04 .65 & 92,4 & 16.01 .70 & 75,1 & 10.05 .69 & 62.8 & 18.01 .66 & 46,1 & 31.03 .71 & 37,2 \\
\hline 11 & 18.12.72 & 144,0 & 18.01 .66 & 127,8 & 05.01 .79 & 120,0 & 25.10 .75 & 109,5 & 25.10 .75 & 89,6 & 13.02 .70 & 74,7 & 31.03 .71 & 62,3 & 24.03.79 & 45,9 & 16.11 .74 & 36,3 \\
\hline 12 & 27.12 .69 & 144,0 & 02.04 .65 & 123,0 & 09.03.79 & 120,0 & 07.03 .80 & 109,5 & 18.12.72 & 88,6 & 19.09 .73 & 73,9 & 05.01 .79 & 61,7 & 12.12 .73 & 45,7 & 10.05 .69 & 35,6 \\
\hline 13 & 08.01 .78 & 144,0 & 10.01 .73 & 121,8 & 29.10.72 & 120,0 & 25.10 .75 & 109,5 & 16.01 .70 & 88,6 & 31.03 .71 & 73,7 & 16.01 .70 & 60,0 & 10.05 .69 & 45,3 & 18.01 .66 & 35,4 \\
\hline 14 & 20.06 .80 & 142,0 & 16.11 .74 & 120,6 & 18.02 .72 & 120,0 & 16.01 .70 & 108,0 & 09.03 .79 & 88,4 & 10.05 .69 & 70,3 & 19.09 .73 & 60,9 & 16.04 .68 & 44,0 & 21.10 .76 & 35,0 \\
\hline 15 & 07.04 .79 & $\mid 138,0$ & 05.01 .79 & 120,6 & 25.10 .75 & 118,0 & 09.03 .79 & 102,6 & 18.01 .66 & 87,0 & 23.11 .68 & 70,0 & 31.08 .68 & 57,8 & 29.10 .72 & 44,0 & 24.03 .73 & 34,5 \\
\hline Média & - & 160,5 & - & 132,5 & - & \begin{tabular}{|l|}
123,7 \\
\end{tabular} & - & 112,8 & - & 97,8 & - & 81,6 & - & 70,0 & - & 53,1 & - & 44,2 \\
\hline
\end{tabular}


Assim, adotando-se a distribuição de freqüência proposta por Gumbel para a série das máximas anuais, podem ser calculados os valores esperados para diferentes períodos de retorno, desde que sejam conhecidos os desvios padrões e médias das intensidades máximas nas diferentes durações.

Os periodos de retorno de 5, 10 e 15 anos foram escolhidos para o estabelecimento da equação 4 , as estimativas das intensidades vez calculado o fator de freqüência $K$ pela equaçăo 4 , as estimativas das intensidades para os diferentes períodos de retorno são feitas $\mathrm{com} \circ$ auxílio da equação 3 . A tabela 4 apresenta esses valores.

0 parâmetro $t_{0}$ encontrado para o período de retorno de 5 anos tem o valor de 13 minutos. O coeficiente de determinaçăo da regressão linear correspondente é 0,99.

Os outros dois parâmetros têm os valores:

$a=937,580$

$\mathrm{n}=0,593$

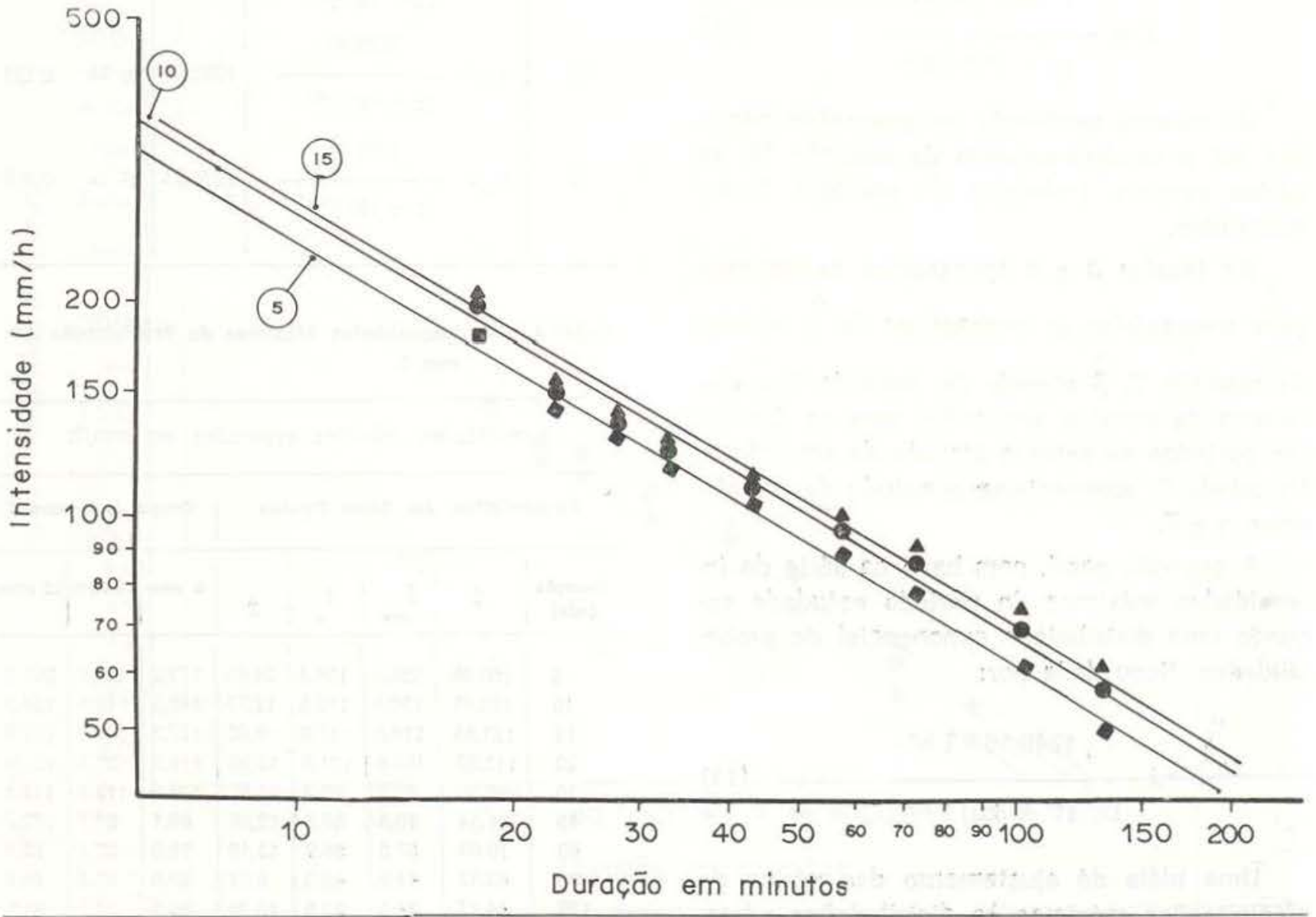

Fig. 2 - Série de máximas anuais método de Guınbel.
A equação resultante para esse período de retorno é

$$
i_{5}=\frac{937,580}{(t+13) 0,593}
$$

As equações correspondentes aos períodos de retorno de 10 a 15 anos são

$$
\begin{aligned}
& i_{10}=\frac{1022,91}{(t+13) 0,593} \\
& i_{15}=\frac{1070,34}{(t+13) 0,593}
\end{aligned}
$$

A condensação desses resultados numa única expressão foi buscada no estudo da relação entre os valores de $a$ e seus correspondentes períodos de retorno. Essa análise é mostrada na tabela 5 . 
TABELA 4 - Séries de Intensidades Máximas Anuais estudadas segundo Gumbel

\begin{tabular}{|c|c|c|c|c|c|c|}
\hline \multicolumn{7}{|c|}{ Intensidades máximas esperadas em $\mathrm{mm} / \mathrm{h}$} \\
\hline \multicolumn{3}{|c|}{$\begin{array}{l}\text { Características } \\
\text { das Séries }\end{array}$} & \multicolumn{3}{|c|}{ Periodo de Retorno } & \multirow{2}{*}{$\begin{array}{l}\text { Volor } \\
\text { Máximo } \\
\text { Obs. }\end{array}$} \\
\hline $\begin{array}{l}\text { Duraşâ。 } \\
\text { (min) }\end{array}$ & $\begin{array}{l}\text { Mádia } \\
\text { (i) }\end{array}$ & $\left|\begin{array}{c}\text { D. Padrăo } \\
\sigma\end{array}\right|$ & 5 anos & 10 enos & 15 anos & \\
\hline 5 & 154,4 & 32,06 & 177,5 & 196,4 & 207,0 & 240,0 \\
\hline 10 & 128,0 & 15,50 & 139,2 & 148,3 & 153,4 & 168,0 \\
\hline 15 & 118,2 & 11,00 & 126,1 & 132,6 & 136,2 & 140,0 \\
\hline 20 & 107,8 & 10,10 & 115,1 & 121,0 & 124,4 & 121,5 \\
\hline 30 & 93,2 & 12,30 & 102,1 & 109,3 & 113,4 & 115,0 \\
\hline 45 & 78,7 & 11.90 & 87,3 & 94,3 & 98,2 & 104,3 \\
\hline 60 & 66.7 & 13,90 & 76,7 & 84,9 & 89,5 & 97,5 \\
\hline 90 & 49,8 & 14,40 & 60,2 & 68,7 & 73,4 & 89,8 \\
\hline 120 & 40,7 & 12,70 & 49,8 & 57,3 & 61,5 & 72,9 \\
\hline
\end{tabular}

A equação geral, com base na série de intensidades máximas anuais estudadas segundo Gumbel, ficou dada por:

$$
i=\frac{772,21 T^{0,121}}{(t+13) 0,593}
$$

$\mathrm{Na}$ mesma seqüência de operações seguidas até o estabelecimento da equação 12 , as séries parciais (máximas do período) foram analisadas.

As tabelas 3 e 6 apresentam as informações necessárias às estimativas de $\hat{\mathrm{i}}_{0}$ através da equação $7, \hat{\beta}$ através da equação 8 e dos valores de eventos esperados para os diferentes períodos de retorno através da equação 9 . $\mathrm{Na}$ tabela 7, apresenta-se o estudo da relação entre a e $T$.

A equação geral, com base na série de intensidades máximas do período estudada segundo uma distrıbuição exponencial de probabilidades, ficou dada por:

$$
i-\frac{1248,56 \cdot T^{0,12}}{(t+20) 0.685}
$$

Uma idéia do ajustamento das séries de precipitações máximas às distribuições adotadas é apresentada na tabela 8 . Para tanto, as séries foram dispostas em ordem decrescente de magnitude. C período de retorno para cada elemento dessas ordenações foi calculado pela relação:

$$
T=\frac{N+1}{N 0}
$$

onde,

No - número de ordem do elemento na seqüência decrescente.

$\mathrm{N}$ - número de anos do período estudado.

TABELA 5 - Estudo da relação $a=c T m$

\begin{tabular}{c|c|c|c}
\hline $\begin{array}{c}T \\
\text { (anos) }\end{array}$ & Equação estabelecida & a & $\begin{array}{l}\text { Parâmetros } \\
\text { encontrados }\end{array}$ \\
\hline 5 & $i_{5}=\frac{937,58}{(t+13)^{0,593}}$ & 937,58 & $c=772,21$ \\
10 & $i_{10}=\frac{1022,91}{(t+13)^{0,593}}$ & 1022,91 & $m=0,121$ \\
15 & $i_{15}=\frac{1070,34}{(t+13)^{0,593}}$ & 1070,34 & $r^{2}=$ \\
& & 0,999 \\
\hline
\end{tabular}

\begin{tabular}{|c|c|c|c|c|c|c|c|}
\hline \multicolumn{2}{|c|}{ Caracteristicas } & \multicolumn{3}{|c|}{ das Séries: } & \multicolumn{3}{|c|}{ Tempo de Retorne $T$} \\
\hline $\begin{array}{c}\text { Duraçăo } \\
\text { (min) }\end{array}$ & $\overline{\mathbf{i}}$ & $\mathbf{i}_{\text {mix }}$ & î & $\hat{\mathbf{B}}$ & 5 anos & 10 anos & 15 anos \\
\hline 5 & 160,48 & 138,0 & 136,4 & 24,09 & 175,2 & 191,9 & 201,6 \\
\hline 10 & 132,48 & 120,6 & 119,8 & 12,73 & 140,3 & 149,1 & 154,3 \\
\hline 15 & 123,65 & 118,0 & 117,6 & 6,05 & 127,3 & 131,5 & 134,0 \\
\hline 20 & 112,82 & 102,6 & 101,9 & 10,95 & 119,5 & 127,1 & 131,6 \\
\hline 30 & 97,80 & 87,0 & 86,2 & 11,57 & 104,8 & 112,8 & 117,5 \\
\hline 45 & 81,54 & 70,0 & 69,2 & 12,36 & 89,1 & 97,7 & 102,7 \\
\hline 60 & 70,03 & 57,8 & 56,9 & 13,10 & 78,0 & 87,1 & 92,4 \\
\hline 90 & 53,13 & 44,0 & 43,3 & 9,78 & 59,0 & 65,8 & 69,8 \\
\hline 120 & 44,17 & 34,5 & 33,8 & 10,36 & 50,5 & 57,7 & 61,9 \\
\hline
\end{tabular}

TABELA 6 - Intensidades Máximas de Precipitação em $\mathrm{mm} / \mathrm{h}$

Intensidades máximas esperadas em $\mathrm{mm} / \mathrm{h}$ 
Os desvios residuais, $s$, em cada série foram calculados pela expressão:

$$
s=\sqrt{\frac{\left(i-i_{o b s}\right)^{2}}{N-1}}
$$

onde,

i - valores estimados pelas equações 12 ou 13

$i_{\text {obs }}$ - valores observados.

As figuras 2 e 3 mostram a aderência dos eventos observados às retas determinadas.

\section{CONCLUSÕES}

- Foram estabelecidas as equações de intensidade-freqüência-duração para a Estação Meteorológica da Reserva Florestal Ducke, situada próxima à cidade de Manaus-AM .
- A utilização de uma amostra não muito grande, 15 anos de observação, não deve prejudicar muito as estimativas das intensidades máximas esperacias, principalmente para períodos de retorno inferiores a 50 anos.

- A escolha entre as duas equações aqui estabelecidas deve apoiar-se no tipo de projeto envolvido. Quando interessa conhecer os valores de intensidades extremas, a série de máximas anuais deve ser utilizada. Na prática, os resultados obtidos nos dois tipos de análise. parecem indicar que não ocorre grande diferença entre as duas estimativas. As séries par. ciais sistematicamente darão valores superio. res, ou, o que é equivalente, períodos de retorno menores para uma mesma intensidade máxima.

- Embora a distribuição de Gumbel seja melhor fundamentada para descrever extremos anuais do que a distribuição exponencial para descrever séries parciais, os dados observados indicam um me!hor ajustamento desta última no caso estudacio.

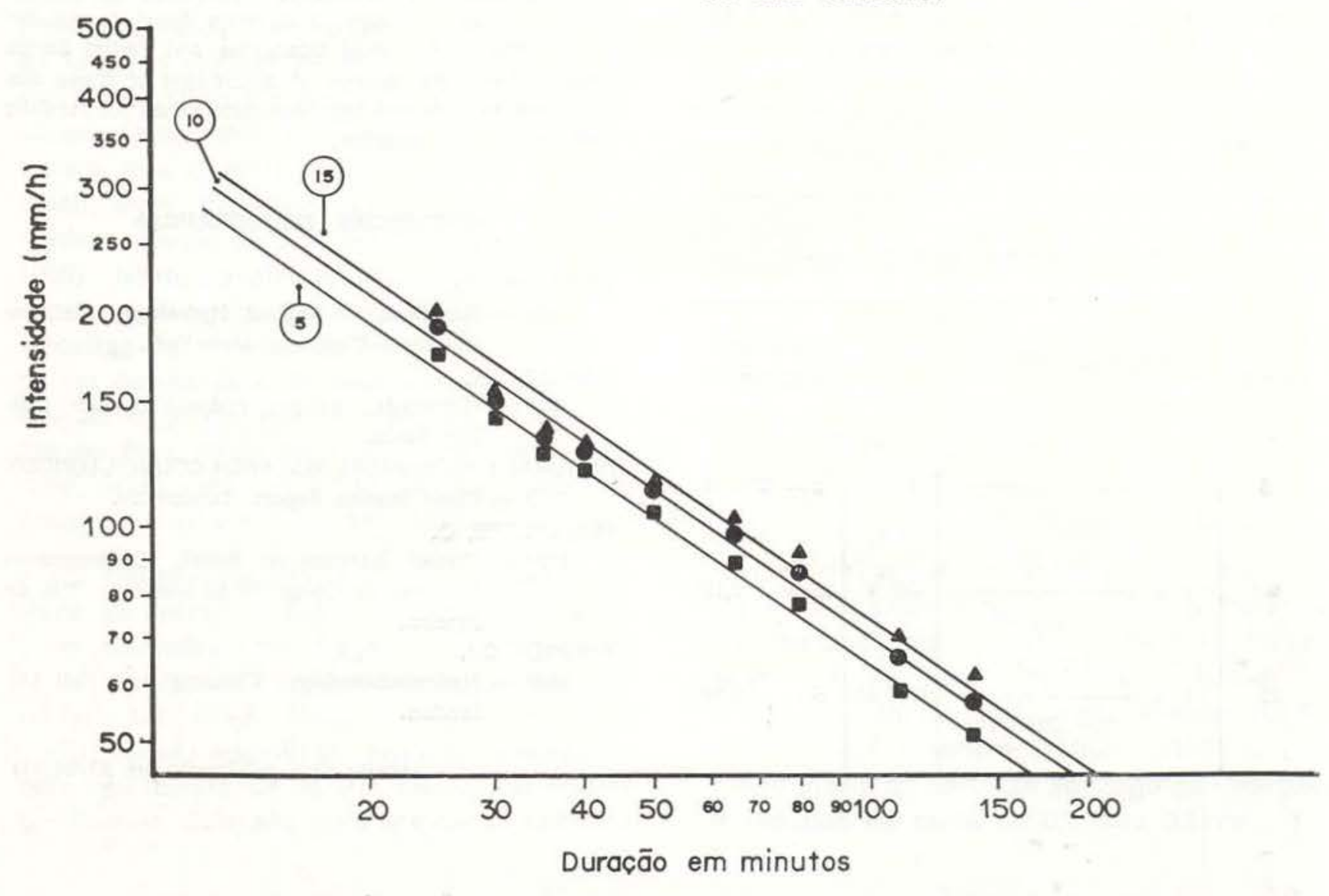

Fig. 3 - Série de duraçāo parcial máximas do período. 
- Os desvios residuais encontrados nas duas séries, $8,2 \mathrm{~mm} / \mathrm{h}$ e $5,1 \mathrm{~mm} / \mathrm{h}$, são relativamente pequenos e mostram a margem de

TABELA 8 - Análise dos erros das estimativas para precipitaçōes com duracões de 10 minutos

\begin{tabular}{|c|c|c|c|c|c|c|c|}
\hline \multicolumn{2}{|c|}{ Caracteristicas } & \multicolumn{3}{|c|}{$\begin{array}{c}\text { Séries de Máximas } \\
\text { Anuais }\end{array}$} & \multicolumn{3}{|c|}{ Séries Parciais } \\
\hline $\begin{array}{l}\text { N.o de } \\
\text { Ordem }\end{array}$ & $\mathrm{T}=\frac{\mathrm{N}+1}{\mathrm{NO}}$ & iobs & i & $\left(i_{\text {obs }}-i\right)_{2}$ & iobs & $\mathbf{i}$ & 1 obs $^{-i)^{2}}$ \\
\hline 1 & 16,00 & 168,0 & 168,2 & 0,04 & 168,0 & 169,5 & 2,25 \\
\hline 2 & 8,00 & 146,4 & 154,7 & 68,89 & 146,4 & 155,9 & 90,25 \\
\hline 3 & 5,33 & 138,0 & 147,3 & 86,49 & 138,0 & 148,5 & 110,25 \\
\hline 4 & 4,00 & 132,0 & 142,3 & 106,09 & 135,0 & 143,5 & 72,25 \\
\hline 5 & 3,20 & 132,0 & 138,5 & 42,25 & 132,0 & 139,7 & 59,29 \\
\hline 6 & 2,67 & 132,0 & 135,5 & 12,25 & 132,0 & 136,7 & 22,09 \\
\hline 7 & 2,29 & 129,0 & 133,0 & 16,00 & 132,0 & 134,2 & 4,84 \\
\hline 8 & 2,00 & 127,8 & 130,8 & 9,00 & 132,0 & 132,0 & 0,00 \\
\hline 9 & 1,78 & 123,0 & 129,0 & 36,00 & 129,0 & 130,2 & 1,44 \\
\hline 10 & 1,60 & 121,8 & 127,3 & 30,25 & 129,0 & 128,6 & 0,16 \\
\hline 11 & 1.45 & 120.0 & 125,8 & 33,64 & 127,8 & 127,0 & 0,64 \\
\hline 12 & 1,33 & 120,0 & 124,5 & 20,25 & 123,0 & 125,7 & 7,29 \\
\hline 13 & 1,23 & 117,0 & 123,3 & 39,69 & 121,8 & 124,6 & 7,84 \\
\hline 14 & 1.14 & 111,0 & 122,2 & 125,44 & 120,6 & 123,4 & 7,84 \\
\hline 15 & 1,07 & 102,0 & 121,3 & 372,49 & 120,6 & 122,5 & 3,61 \\
\hline & & & 8,2 & & & 5, & h \\
\hline
\end{tabular}

TABELA 7 - Estudo da relação $a=\mathrm{cTm}$

\begin{tabular}{|c|c|c|c|c|c|}
\hline $\begin{array}{c}T \\
\text { (anos) }\end{array}$ & \multicolumn{2}{|c|}{ Equação estabelecida } & a & \multicolumn{2}{|c|}{$\begin{array}{l}\text { Parâmetros } \\
\text { encontrados }\end{array}$} \\
\hline \multirow{2}{*}{5} & \multirow{2}{*}{$i=$} & 1513,16 & \multirow{2}{*}{1513,16} & \multicolumn{2}{|c|}{$c=1248,560$} \\
\hline & & $(t+20)^{0,605}$ & & $\mathrm{c}=$ & \\
\hline \multirow{2}{*}{10} & \multirow{2}{*}{$i=$} & 1647,29 & \multirow[b]{2}{*}{1647,20} & \multirow[b]{2}{*}{$m=$} & \multirow[b]{2}{*}{0,120} \\
\hline & & $(t+20)^{0,605}$ & & & \\
\hline \multirow{2}{*}{15} & \multirow{2}{*}{$i=$} & 1725,28 & \multirow{2}{*}{1725,28} & \multirow{2}{*}{$r^{2}=$} & \multirow{2}{*}{0,999} \\
\hline & & $(t+20)^{0,635}$ & & & \\
\hline
\end{tabular}

erro que deve ser esperada nas estimativas de chuvas com durações de 10 minutos.

- Os projetos de obras hidráulicas na cidade de Manaus podem apoiar-se nas informações sobre intensidades máximas prováveis fornecidas pelas equaçōes aqui estabelecidas.

\section{AgRAdeCIMENTOS}

Os autores agradecem às Auxiliares Técnicas Valdelice Pereira da Silva, Maria Solange da Graça Pinheiro e Maria Aparecida da Silva Gomes pelo apoio prestado na preparação dos dados pluviográficos.

\section{SUMMARY}

In this project, the relationship between intensity, frequency and duration of rainfall was studied, in the period since 1965 at the Principal Weather Station of the Ducke Forest Reserve belonging to the Instituto Nacional de Pesquisas da Amazônia, kilometer 26 of the Torquato Tapajós highway.

The frequency distribution proposed by Gumbel and an exponential distribution were fitted to the Series of Maximum Annual Intensities and Partial Series were formed. The degree of adjustment of these distributions to observed data was determined for rainfalls with 10 minutes duration.

\section{REFERENCIAS BIBLIOGRÁFICAS}

CHOW, Ven Te

1964 - Handbook of Applied Hydrology. McGrawHill Book Company. New York.

GARCEZ, L.N.

1974 - Hidrologia. Editora Ec'dgard Blücher Ltda. São Paulo.

NATURAL ENVIRONMENT RESEARCH COUNCIL-LONDON 1975 - Flood Studies Report. London. 2v.

PFAFSTETTER, $\mathrm{O}$.

1957 - Chuvas Intensas no Brasil. Departamento Nacional de Obras de Saneamento. Rio de Janeiro.

WIESNER, C.J.

1970 - Hydrometeorology. Chapman and Hall Ltd. London.

(Aceito para pub'icação em 23/04/81) 